\title{
Compact Photonic Integrated Chip for Tunable Microwave Generation
}

\author{
Chen-Yu Chien, Yen-Hua Lo, Yu-Chang Wu, Shun-Chieh Hsu, Hung-Ruei Tseng, \\ Chien-Chung Lin, Senior Member, IEEE
}

\begin{abstract}
A tunable microwave generation is demonstrated by monolithically integrated photonic chip, which combines a mirror section and two distributed feedback (DFB) lasers. The focus ion beam technology was employed to achieve high-quality air/semiconductor slab for the mirror. Two DFB lasers (named slave and master lasers, respectively) can operate in a single fundamental mode when they are individually pumped. A microwave signal with the differential frequency between the two lasers can be obtained through optical heterodyning. A tunable microwave signal up to $25.5 \mathrm{GHz}$ can be generated by adjusting the injected currents of the two DFB lasers. A four-wave mixing spectrum was measured and the linewidth of the generated radio frequency signal is $1.9 \mathrm{MHz}$.
\end{abstract}

Index Terms-Semiconductor laser, microwave generation, radio frequency photonics, heterodyne.

\section{INTRODUCTION}

$\mathbf{T}$ HE MICROWAVE generation by photonic devices is very important in modern communication systems [1]. From all-optical clock generation [2], ultra-high speed modulation [3], to radio over fiber (RoF) systems [4], many applications require a highly stable microwave source based on photonic devices. It is particularly useful in the radio over fiber (RoF) scheme where a compatible microwave source can simplify the complicated mobile networks and fully utilize the existing fiber network infrastructure [4]. To implement a reliable and compact microwave generation, one of the promising solutions is using two monochromatic optical signals to generate the beating radio-frequency (RF) signals (also called optical heterodyne) [3], [5]-[7]. Compared to the heterodyne system made by the discrete components, the photonic integrated circuit (PIC) design is much more advantageous in terms of size and efficiency. In previous research, integration of two or multiple optoelectronic components into one chip to realize such function was demonstrated [2], [8]-[14]. However, most of them involved several re-growth steps (such as Y-branch passive waveguide regrowth) or complex fabrication processes. On the other hand, due to the

Manuscript received November 23, 2013; revised December 19, 2013; accepted January 2, 2014. Date of publication January 9, 2014; date of current version February 5, 2014. This work was supported by the National Science Council of Taiwan under Grants NSC-99-2221-E-009-052-MY3, NSC 102-2120-M-110-005, and NSC 101-2221-E-009-046-MY3.

The authors are with the Institute of Photonic System, National Chiao-Tung University, Tainan 700, Taiwan (e-mail: chienchunglin@faculty.nctu.edu.tw).

Color versions of one or more of the figures in this letter are available online at http://ieeexplore.iee.org.

Digital Object Identifier 10.1109/LPT.2014.2298983 progress in micro/nano-fabrication, artificial structures such as air slot and dry-etched distributed Bragg reflectors (DBRs) became available for integration or performance enhancement [15]-[19]. These structures, often made by dry etch, can provide a post-epitaxial adjustment to strengthen device performance, and also eliminate tedious dielectric deposition steps. Single mode lasers and wavelength tunable lasers were demonstrated previously using this idea [15]-[17], [20]. The dry etch process needs to rely on precise photolithography which can be difficult if the structure is in sub-micron regime and the etch step has to be carefully included in the process. One of the nano-scale fabrication technologies which can alleviate these concerns is called focused ion beam (FIB). This technique utilizes high energy particle beams to etch off semiconductor material. With high precision and post-process adjustment capability, FIB is widely used in transmission electron microscopy (TEM) sample preparation, post-process device diagnosis, and mirror-like surface fabrication [21]. Previously we reported the idea of utilizing FIB technology and demonstrate an air/semiconductor distributed Bragg reflector (DBR) design to incorporate two distributed feedback lasers (DFB) in one monolithic platform and provide a tunable output of microwave signals [22]. In this letter, the benefit of such design is fully investigated and the detailed RF result is presented. The integration scheme is simple and the robust DFB design ensures single mode laser operation to acquire a good microwave output. The final dual-wavelength operation of the chip can produce a beating note between the two optical lights and generate a narrow-linewidth microwave signal. In the following text, both the design and the experimental results will be elucidated.

\section{Design of Distributed Bragg Reflectors}

The device schematic was shown in Fig. 1. In a two-section DFB laser chip, an air/semiconductor DBR structure is placed in the center section between the two lasers. The front laser is called the slave laser, and the backside one is called the master laser. With the help of focused ion beam (FIB), specifically defined pattern can be etched onto the semiconductor ridge of the lasers and form air/semiconductor slab structure. The FIB etch usually cut through the waveguide core to generate enough refractive index contrast for the mirror, and the electrical isolation as well. The role of DBR, in addition to serve as the common mirror of both lasers, is also regarded as the optical coupler which introduces the optical field of the master laser into the slave laser and vice versa. 


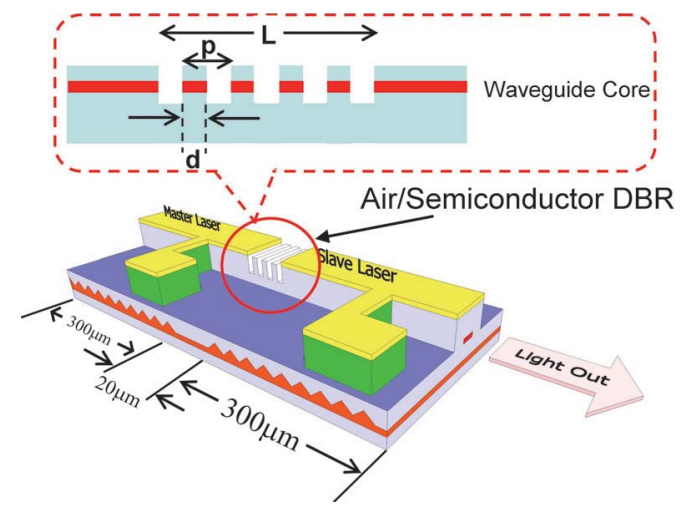

Fig. 1. The schematic diagram of a compact two-section laser. The front laser is called the slave laser and the backside one is the master. The air/semiconductor slabs are placed in the center for highly reflective mirror purpose. The important parameters for the mirror design are also shown in the plot.

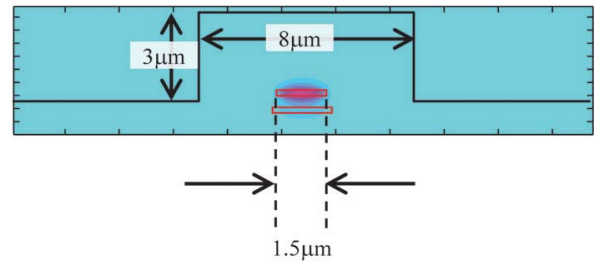

Fig. 2. The cross-section view of the field distribution of the fundamental mode calculated by finite difference technique.

If the reflectivity of the DBR is large (as in our case), the coupling between the two lasers is weak and a stable operation can be expected. However, if the reflectivity is too low, the two DFB sections are no longer isolated from each other and can be viewed as a composite coupled-cavity, in which the original dual-mode heterodyne might not work at all. The period of each pair, $p$, and the semiconductor layer width, $d$, (both shown in the Fig. 1) are two important parameters to be controlled in our design. One less obvious constraint is the total length of this DBR section, which is denoted as L in Fig. 1. The physical dimensions of the integrated chip are as following: the cavity length of slave and master lasers: $300 \mu \mathrm{m}$; the total length of FIB-able section: $20 \mu \mathrm{m}$; ridge width: $8 \mu \mathrm{m}$; active mesa: $1.5 \mu \mathrm{m}$. The limited length on FIB section will put constraint on the DBR design.

The basic ridge waveguide device is composed of two layers: the active wave-guiding layer (plus grating) and the surrounding passive material. The device is in a 3-dimensional structure, and to simulate this structure properly, several methods need to be considered to generate suitable evaluations. First, a finite difference method is applied to calculate the fundamental mode of the waveguide formed by the active layer and its surrounding InP material [23]. The results can be shown in Fig. 2. After the intensity profile of the fundamental mode is found, we can determine the effective index of the overall waveguide, which is 3.1896 at $\lambda=1550 \mathrm{~nm}$ [23], [24]. Other methods, such as 3-dimensional finite-difference timedomain (3D FDTD) simulation, can also perform similar calculations at higher precision but with requirement of greater computer resources [18]. The DBR reflectivity can be then

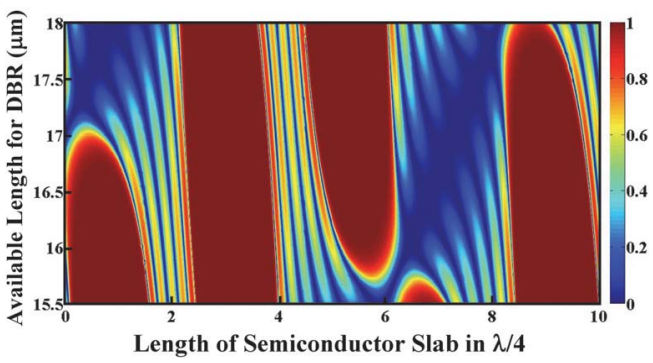

Fig. 3. The calculated reflectivity of air/semiconductor DBR design under different total length constraint (Y-axis) and length of each semiconductor slab in quarter wavelengths. The color bar indicates the calculated reflectivity.

calculated by transmission matrix method [23]. The total DBR length $(\mathrm{L})$ is confined within $18 \mu \mathrm{m}$ such that FIB area is at least $1 \mu \mathrm{m}$ away from the lasers. The total number of DBR pairs is fixed at 10.5 due to electrical isolation and optical mode concerns. The Fig. 3 shows our calculation of reflectivity under the effective index model. The wide range of high reflectivity (nearly 1) can be found in our design, and even though this is a highly idealized calculation, it means our design can be robust against possible process variation. For example, a variation between 2.7 and 3.3 quarter-wavelengths can still produce nearly $100 \%$ of reflectivity. So a $10 \%$ etching error, which is achievable in current process, can be tolerated in this design and the reflectivity is not inflicted seriously.

\section{Device Fabrication}

The epitaxial layers were grown on a n-type InP substrate by a MOCVD system, and a InGaAsP multiple-quantum-well epitaxial layer is the gain medium. After MOCVD growth, mesa etch steps and a subsequent blocking-layer regrowth were carried out to form the buried-heterostructure (BH). The grating pitch of each laser is designed for $1550 \mathrm{~nm}$ Bragg wavelength, and manufactured through direct E-beam lithography. The wafer was then taken through regular processing steps (including metalization, ridge etch, and deposition of silicon nitride for device isolation, etc.) to have the basic device feature finished. After wafer processing, we further thin the wafer down, dice and bond the laser chip onto a ceramic submount.

The final step is the focused ion beam (FIB) etch, which was accomplished in a vacuum chamber and a $\mathrm{Ga}$ ion beam with $30 \mathrm{keV}$ (the equivalent current varying from 1 to $5 \mathrm{nA}$ ) was used to carve the ridge between the two laser sections. We optimized the FIB etch to prevent the air/semiconductor interface from unwanted roughness, which can be detrimental for laser operation. The etch rate of FIB procedure is about $1.5 \mu \mathrm{m} / \mathrm{min}$, and the etch pattern can be pre-defined by software. We use 3 quarters of Bragg wavelength for the DBR layer thickness and the width of the semiconductor and air gap is $360 \mathrm{~nm}$ and $1160 \mathrm{~nm}$, respectively. The final air/semiconductor DBR can be seen in Fig. 4. Different etch depths were tried: $1 \mu \mathrm{m}, 3 \mu \mathrm{m}$, and $5 \mu \mathrm{m}$. From the measured free-running laser spectrum, we decided the optimal depth is $5 \mu \mathrm{m}$. For shallower depth (like $1 \mu \mathrm{m}$ and $3 \mu \mathrm{m}$ ), the laser can only work in multiple longitudinal mode, and the electrical resistance between master and slave lasers is too low. 


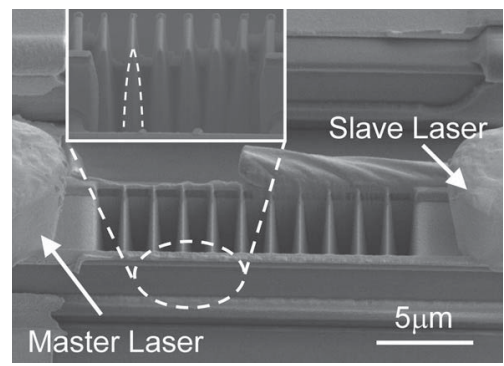

Fig. 4. The air/semiconductor DBR after FIB process. The inset is the cross-section view of the etched air/semiconductor DBR from the same viewing angle. The cross-sectional profile of the DBR in the inset was revealed after the sidewall structure was etched away. The dot line is the etched air/semiconductor DBR profile.

With $5 \mu \mathrm{m}$, both strong side mode suppression and electronic isolation can be satisfied. The direct resistance measurement between two anodes is about 200 ohms. A further inspection on the sidewall profile of SEM reveals that the actual etch depth is around $5.15 \mu \mathrm{m}$, and the width of semiconductor portion of the mirror is around $0.96 \mu \mathrm{m}$ due to the slant profile of FIB. An example of side wall profile can be seen in the inset of Fig. 4.

\section{RESUlts AND Discussion}

The uncoated regular DFB is prone to multimode operation. The introduction of DBR at one end ensures of the single mode operation of each DFB section which is important for the microwave generation. The optical spectrum and LI characteristics of an individually driven DBR DFB laser are shown in Fig. 5. A pure single mode with side-mode- suppression ratio (SMSR) larger than 40dB was measured, and a good laser LI in free-space (no fiber coupling) with $6 \mathrm{~mA}$ of threshold current proves this FIB etch is successful.

To take the RF signal data, a three probe station was needed to bias the two lasers at $\mathrm{I}_{\text {slave }}$ and $\mathrm{I}_{\text {master }}$ simultaneously. A bare fiber tip was placed close to the chip facet to collect the emitted light. The coupled light was directed into either optical spectrum analyzer (OSA) for laser emission spectrum or a high-speed photodetector (NewFocus $1414,25 \mathrm{GHz}$ IR photodetector) and an Agilent 8564E ESA for further readout of RF signals. The combined optical spectrum from the slave laser facet can be seen in Fig. 6(a), and the microwave spectrum of the beating note can be extracted from the photo-currents of the highspeed detector, shown in Fig. 6(b). In our test, the bias current of the slave laser $\left(\mathrm{I}_{\text {slave }}\right)$ is fixed at $70 \mathrm{~mA}$ while the input current of the master laser $\left(\mathrm{I}_{\text {master }}\right)$ is continuously increased and thus its emission wavelength will be red-shifted correspondingly. This gradual increase of the master laser's wavelength, when sweeping through the slave laser's peak, can generate a continuously tunable microwave signal via optical heterodyne, and the frequency of this signal can be determined by the separation of the two peaks, called the detuning frequency $(\Delta \mathrm{f})$, and calculated as:

$$
\Delta f=c\left(1 / \lambda_{\text {master }}-1 / \lambda_{\text {slave }}\right),
$$

where $\mathrm{c}$ is the speed of light, and $\lambda_{\text {master }}$ and $\lambda_{\text {slave }}$ are the wavelengths of emission from the two lasers. As been

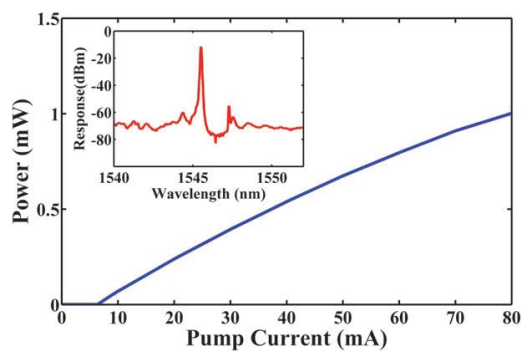

Fig. 5. A typical free-space LI of the air/semiconductor DBR DFB laser and its spectrum (inset).

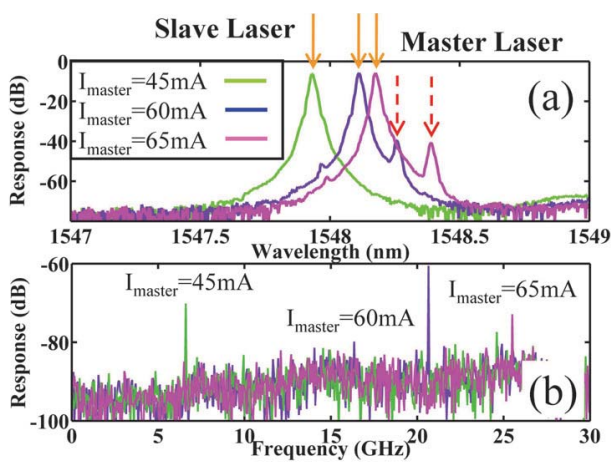

Fig. 6. (a) The combined optical spectrum when both slave and master lasers are on. The slave laser pumping current was kept at $70 \mathrm{~mA}$. The emission wavelengths of the master laser are changed by different injection current ( $\mathrm{I}_{\text {master }}$ ) and can be seen at the dot arrow positions. At $\mathrm{I}_{\text {master }}=45 \mathrm{~mA}$, the two laser's emission wavelengths are so close that the OSA can not resolve. (b) The measured RF spectrum of the DBR integrated DFB lasers at the same bias currents as (a).

published before, when the injected power into the slave laser is weak, the slave laser will be in either a chaotic state, a period-one state or a four-wave mixing (FWM) state depending on the $\Delta \mathrm{f}[25]$. From the optical spectrum in Fig. 6(a), our slave laser operates in the FWM state when both lasers are on. Because the actual reflectivity of the DBR is not $100 \%$, a mutual injection between the slave and master lasers happens and the similar FWM spectrum can be observed from the other facet of the master laser. These externally injected photons are, however, much less compared to the ones in the cavity such that the single mode operation of the laser can be maintained. The origin of the FWM feature rises from the fluctuation of carrier number in the laser due to the injected power, and the frequency separation between the satellite peaks and the main one $(\Delta f)$ in the optical domain [Fig. 6(a)] can directly correspond to the RF frequencies in Fig. 6(b) [5], [7], [25], [26]. From our measurement, a series of RF signal can be detected up to $25.5 \mathrm{GHz}$. This RF measurement is, however, limited by the bandwidth of our photodetector, and thus we did not fully explore the entire range. From the wavelength differences of the two lasers, one can deduct that an RF signal as high as $50 \mathrm{GHz}$ could be generated. A further separation, however, won't be able to acquire enough power via FWM and thus might not be useful.

The linewidth of the RF signal is another important parameter to be identified. In most applications, it is desirable to have a narrow linewidth signal to start with. In the optical heterodyne, if the line shape of the laser emission spectrum is Lorentzian, the generated signal will also be a Lorentzian 


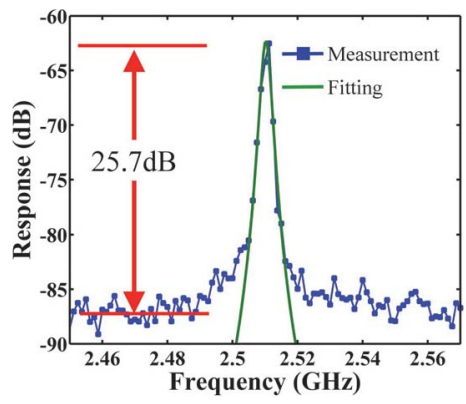

Fig. 7. The detailed RF spectrum of the generated signal. The blue dots are the measurement. The fitted curve (green) indicates a 3dB-linewidth of $1.883 \mathrm{MHz}$ for the microwave signal generated by the integrated device.

and carry the summation of the linewidths of each laser [14]. We reduce the resolution bandwidth to $300 \mathrm{kHz}$ in the spectrum analyzer and acquire the detailed data points of the generated RF signal in this DBR type of devices. As shown in the Fig. 7, the measured linewidth is about $1.9 \mathrm{MHz}$, which is reasonable for a DFB laser with sub-mega hertz linewidth. An $25.7 \mathrm{~dB}$ peak-to-noise floor also marks the superiority of the generated RF signal.

\section{CONCLUSION}

In conclusion, a monolithically integrated dual-wavelength DFB laser was fabricated for the microwave generation. With FIB technology, a nano-scale DBR structure can be created in between the two lasers and provide high reflectivity for single mode operation. When two lasers are electrically pumped together, the difference of the two emission wavelengths can be used for optical heterodyning and a high quality RF beating tone can be detected by a high speed photodiode. A tunable microwave signal can be realized up to $25.5 \mathrm{GHz}$, limited by the bandwidth of our photodiodes. The linewidth of this mixed signal is less than $2 \mathrm{MHz}$ and other schemes could be considered for its further improvement. Many solutions require good optical isolation between two lasers, which might need to be carefully evaluated before adapting into our DBR design. This integrated device has the capability to deliver a high output power and small footprint module and it can be a promising candidate for the future generation of compact microwave source.

\section{ACKNOWLEDGMENT}

Authors would like to thank Prof. H.-C. Kuo, Prof. J.-H. Chen of NCTU, Dr. Y.-J. Cheng of SINICA in Taiwan and Santur Corporation, Inc. USA for their technical supports.

\section{REFERENCES}

[1] A. J. Seeds and K. J. Williams, "Microwave photonics," J. Lightw. Technol., vol. 24, no. 12, pp. 4628-4641, Dec. 2006.

[2] U. Feiste, D. J. As, and A. Erhardt, " $18 \mathrm{GHz}$ all-optical frequency locking and clock recovery using a self-pulsating two-section DFB laser," IEEE Photon. Technol. Lett., vol. 6, no. 1, pp. 106-108, Jan. 1994.

[3] H.-K. Sung, "Strong optical injection locking of edge-emitting lasers and its applications," Ph.D. dissertation, Dept. Electr. Eng. Comput. Sci., Univ. California Berkeley, Berkeley, CA, USA, Tech. Rep. no. UCB/EECS-2006-107, 2006.
[4] C. T. Lin, et al., "Hybrid optical access network integrating fiber-to-thehome and radio-over-fiber system," IEEE Photon. Technol. Lett., vol. 19, no. 8, pp. 610-612, Apr. 15, 2007.

[5] J.-M. Liu and T. B. Simpson, "Four-wave mixing and optical modulation in a semiconductor laser," IEEE J. Quantum Electron., vol. 30, no. 4, pp. 957-965, Apr. 1994.

[6] L. Goldberg, H. F. Taylor, J. F. Weller, and D. M. Bloom, "Microwave signal generation with injection-locked laser diodes," Electron. Lett., vol. 19, no. 13, pp. 491-493, Jun. 1983.

[7] J. Yao, "Microwave photonics," J. Lightw. Technol., vol. 27, no. 3, pp. 314-335, Feb. 1, 2009.

[8] A. Tauke-Pedretti, et al., "Mutual injection locking of monolithically integrated coupled-cavity DBR lasers," IEEE Photon. Technol. Lett., vol. 23, no. 13, pp. 908-910, Jul. 1, 2011.

[9] H.-K. Sung, T. Jung, M. C. Wu, D. Tishinin, K. Y. Liou, and W. T. Tsang, "Optical generation of millimeter-waves using monolithic sideband injection locking of a two-section DFB laser," in Proc. 16th LEOS Annu. Meeting, vol. 2. Tucson, AZ, USA, 2003, pp. 1005-1006.

[10] N. Kim, et al. "Monolithic dual-mode distributed feedback semiconductor laser for tunable continuous-wave terahertz generation," Opt. Express, vol. 17, no. 16, pp. 13851-13859, Aug. 2009.

[11] R. K. Price, V. B. Verma, K. E. Tobin, V. C. Elarde, and J. J. Coleman, "Y-branch surface-etched distributed Bragg reflector lasers at $850 \mathrm{~nm}$ for optical heterodyning," IEEE Photon. Technol. Lett., vol. 19, no. 20, pp. 1610-1612, Oct. 15, 2007.

[12] J. Huang, C. Sun, B. Xiong, and Y. Luo, "Y-branch integrated dual wavelength laser diode for microwave generation by sideband injection locking," Opt. Express, vol. 23, no. 17, pp. 20727-20734, Nov. 2009.

[13] C. Kim, et al., "Optical microwave/millimeter-wave links using direct modulation of two-section gain-coupled DFB lasers," IEEE Photon. Technol. Lett., vol. 17, no. 8, pp. 1734-1736, Aug. 2005.

[14] C. Laperle, M. Svilans, M. Poirier, and M. Tetu, "Frequency multiplication of microwave signals by sideband optical injection locking using a monolithic dual-wavelength DFB laser device," IEEE Trans. Microw. Theory Tech., vol. 47, no. 7, pp. 1219-1224, Jul. 1999.

[15] J. P. Engelstaedter, B. Roycroft, F. Peters, and B. Corbett, "Wavelength tunable laser using an interleaved rear reflector," IEEE Photon. Technol. Lett., vol. 22, no. 1, pp. 54-56, Jan. 1, 2010.

[16] Q. Lu, et al., "Single mode lasers based on slots suitable for photonic integration," Opt. Express, vol. 19, no. 26, pp. B140-B145, Dec. 2011.

[17] T. Yu, L. Zou, L. Wang, and J.-J. He, "Single-mode and wavelength tunable lasers based on deep-submicron slots fabricated by standard UV-lithography," Opt. Express, vol. 20, no. 15, pp. 16291-16299, Jul. 2012.

[18] B. Docter, et al., "Discretely tunable laser based on filtered feedback for telecommunication applications," IEEE J. Sel. Topics Quantum Electron., vol. 16, no. 5, pp. 1405-1407, Sep. 2010.

[19] H. C. Kim, H. Kanjo, S. Tamura, and S. Arai, "Narrow-stripe distributed reflector lasers with first-order vertical grating and distributed Bragg reflectors," IEEE Photon. Technol. Lett., vol. 15, no. 8, pp. 1032-1034, Aug. 2003.

[20] M. Ariga, et al., "Low threshold GaInAsP lasers with semiconductor/air distributed Bragg reflector fabricated by inductively coupled plasma etching," Jpn. J. Appl. Phys., vol. 39, no. 6A, pp. 3406-3409, Jun. 2000.

[21] S. Matsui and Y. Ochiai, "Focused ion beam applications to solid state devices," Nanotechnology, vol. 7, no. 3, pp. 247-258, Sep. 1996.

[22] C. Lin, C. Lin, C. Chien, and H. Kuo, "Tunable microwave generation by photonic integrated distributed feedback lasers," in Proc. Conf. CLEO, San Jose, CA, USA, May 2012, pp. 1-2, paper JW2A.104.

[23] L. A. Coldren and S. W. Corzine, "Dielectric waveguides," in Diode Lasers and Photonic Integrated Circuits, Hoboken, NJ, USA: Wiley, 1995, ch. 7.

[24] M. J. Adams, "Guides of rectangular cross section," in An Introduction to Optical Waveguides, Hoboken, NJ, USA: Wiley, 1981, ch. 6.

[25] S.-C. Chan, S.-K. Hwang, and J.-M. Liu, "Period-one oscillation for photonic microwave transmission using an optically injected semiconductor laser," Opt. Express, vol. 15, no. 22, pp. 14921-14935, Oct. 2007.

[26] K. Inoue, T. Mukai, and T. Saitoh, "Nearly degenerate four-wave mixing in a traveling-wave semiconductor laser amplifier," Appl. Phys. Lett., vol. 51, no. 14, pp. 1051-1053, Oct. 1987. 